\title{
WWP2 Gene
}

National Cancer Institute

\section{Source}

National Cancer Institute. WWP2 Gene. NCI Thesaurus. Code C95562.

This gene is involved in ubiquitination. 